\title{
Efficient Heuristic for Resource Allocation in Zero-forcing MISO LTE-Advanced Systems with Minimum Rate Constraints
}

\author{
Diego Perea-Vega, , Jean-François Frigon, , and André Girard,
}

\begin{abstract}
4G wireless access systems require high spectral efficiency to support the ever increasing number of users and data rates for real time applications. Multi-antenna OFDM-SDMA systems can provide the required high spectral efficiency and dynamic usage of the channel, but the resource allocation process becomes extremely complex because of the augmented degrees of freedom. In this paper, we propose two heuristics to solve the resource allocation problem that have very low computational complexity and give performances not far from the optimal. The proposed heuristics select a set of users for each subchannel, but contrary to the reported methods that solve the throughput maximization problem, our heuristics consider the set of real-time (RT) users to ensure that their minimum rate requirements are met. We compare the heuristics' performance against an upper bound and other methods proposed in the literature and find that they give a somewhat lower performance, but support a wider range of minimum rates while reducing the computational complexity. The gap between the objective achieved by the heuristics and the upper bound is not large. In our experiments this gap is $10.7 \%$ averaging over all performed numerical evaluations for all system configurations. The increase in the range of the supported minimum rates when compared with a method reported in the literature is $14.6 \%$ on average.
\end{abstract}

\section{INTRODUCTION}

With the ubiquitous use of smart phones, tablets, laptops and Real-Time (RT) applications, traffic demand on the wireless access network is increasing exponentially [1]. In contrast, mobile subscription prices have flattened in the last years due to competition and maturity of the market [2]. Therefore, there is a need to design systems that support high data rates traffic with strict time deadlines, and concurrently optimize the system resources to make deployments economically profitable. 
One of the key system design parameters in $4 \mathrm{G}$ wireless access networks is spectral efficiency. Using spatial, user and frequency diversity techniques in a multi-user Multiple Input Multiple Output (MIMO)-OFDMA system, provides us with a high spectral efficiency. These systems are proposed in current 4G standards, such as Long Term Evolution (LTE) and IEEE 802.16 [3], [4]. However, when increasing the degrees of freedom for transmission, a price has to be paid. Multi-antenna systems require more hardware and software resources to process the multiple spatial layers. In addition, the Resource Allocation (RA) process becomes much more complex because we have many more possibilities from which to choose.

The problem we deal with in this paper is the design of efficient RA algorithms that provide us with solutions not too far from the optimal, for a Zero-Forcing (ZF) Multiple Input Single Output (MISO)-OFDMA system supporting minimum rates. This RA problem is a nonlinear, non-convex integer program, which makes it almost impossible to solve directly for any realistic number of subchannels, users and antennas. For this reason, most research work focuses on developing heuristic algorithms. It is also important to benchmark the performance of these heuristic algorithms. In [5], a dual method is proposed to find a near-optimal solution to the sum rate maximization problem with minimum rate constraints. It requires an enumeration of all Spatial Division Multiple Access (SDMA) sets, which prevents the method to be implemented efficiently, but it provides us an off-line method useful for heuristic benchmarking.

Several heuristic methods have been used to solve the RA problem for OFDMA-SDMA systems with both RT and non-Real Time (nRT) traffic. In [6], the objective is to maximize the sum of the user rates subject to per-user minimum rate constraints that model the priority assigned to each user at each frame. The optimization problem is solved approximately for each frame by minimizing a cost function representing the increase in power needed when increasing the number of users or the modulation order. The advantages of this approach are that it handles user scheduling and RA together and supports RT and nRT traffic. Its weaknesses are that no comparison is made against a near-optimal solution and the method used to determine user priorities at every frame is very complex. In [7], both RT and nRT traffic are supported. Priorities are set according to the remaining deadline time for RT users and to the difference between the achieved rate and the desired rate required for nRT users. Comparisons against the algorithm in [6] show that the packet drop rate for RT users and the algorithm's complexity 
are significantly reduced. However, as in [6], a performance comparison with a near-optimal solution is not provided.

In [8], a heuristic algorithm is proposed for the sum rate maximization problem with proportional rates among the user data rates, i.e., the ratio among allocated user rates is predetermined. The criteria used to form user groups includes semi-orthogonality as in [7], but also fairness through proportional rate constraints. This method is extended to include hard minimum rates in [9]. There is no reported method to evaluate the accuracy of these heuristics, except by comparing them with each other.

In the heuristic method [10], the objective is the weighted sum rate maximization under a total power constraint. The user weights are updated at each frame to include different fairness criteria. When compared to [8] the performance is better, but Jain's fairness index is lower. The sequential user selection to swap users require the channel matrix inversion of all candidates, similarly to [9], which creates a computational burden. In addition, for RT users the method introduces delays by first detecting that the user rates are lower than expected, and then adjusting the user weights, i.e. no hard rate constraints are considered.

Our work differs from previously reported methods because we consider hard minimum rate constraints for real-time users, which is advantageous in terms of delay and QoS compliance. We propose two efficient heuristic methods with much lower computational complexity than the methods proposed in the literature. The computational complexity reduction is several orders of magnitude depending on the algorithm used and the problem parameters. We compare the proposed heuristics performance against the near-optimal solution proposed in [5] and find that the performance obtained is within $10.7 \%$ of the optimal averaging over all performed numerical evaluations. In addition, the proposed heuristics increase the range of the supported minimum rates when compared with the method proposed in [9]. For the same case above, the increase in the rate range is $14.6 \%$ on average. This increase is achieved by considering the rate constraint dual variables in the user power allocation stage.

The paper is organized as follows, in section II, we mathematically formulate the problem we want to solve: for a given time slot, find the user selection and beamforming vectors that maximize a linear utility function of the user rates, given a total transmit power constraint and minimum rate constraints for RT users. In section III, we propose two heuristic methods to solve the problem more efficiently. We compare their performance against the upper bound and against 
other method proposed in the literature in section IV. Finally in section V, we summarize the main findings and state our conclusions.

\section{PROBLEM FORMULATION AND DUAL-BASED NEAR-OPTIMAL METHOD}

We consider the resource allocation problem for the downlink transmission in a multi-carrier multi-user multiple input single output (MISO) system with a single base station (BS). There are $K$ users, some of which have RT traffic with minimum rate requirements while the others have nRT traffic that can be served on a best-effort basis. The BS is equipped with $M$ transmit antennas and each user has one receive antenna. The system's available bandwidth $W$ is divided into $N$ subchannels whose coherence bandwidth is assumed larger than $W / N$, thus each subchannel experiences flat fading. In the system under consideration the BS transmits data in the downlink direction to different users on each subchannel by performing linear beamforming precoding. At each OFDM symbol, the BS changes the beamforming vector for each user on each subchannel to maximize a weighted sum rate. We assume that we use a channel coding that reaches the channel capacity.

The BS transmits on each subchannel $n$, the signal vector $\mathbf{x}_{n}=\sum_{k} \mathbf{w}_{n, k} s_{n, k}$, where $\mathbf{w}_{n, k} \in$ $\mathbb{C}^{M \times 1}$ and $s_{n, k} \in \mathbb{C}$ are, respectively, the beamforming vector and the information symbol for user $k$ on subchannel $n$. The symbols $s_{n, k}$ are assumed to be independent and follow the $\mathcal{C} \mathcal{N}(0,1)$ distribution. A power constraint $\sum_{n, k}\left\|\mathbf{w}_{n, k}\right\|^{2} \leq \check{P}$ is also imposed. The signal received at user $k$ on subchannel $n$ is then given by

$$
y_{n, k}=\mathbf{h}_{n, k} \mathbf{w}_{n, k} s_{n, k}+\sum_{j \neq k} \mathbf{h}_{n, k} \mathbf{w}_{n, j} s_{n, j}+z_{n, k} .
$$

where $\mathbf{h}_{n, k} \in \mathbb{C}^{1 \times M}$ is the channel row $M$-vector between the BS and user $k$ on subchannel $n$, and $z_{n, k} \sim \mathbb{C N}(0,1)$ is the white additive noise at the receiver. The second term in Eq. (1) corresponds to the inter-user interference. To simplify the RA problem, we assume that the beamforming vectors are chosen according to the zero forcing (ZF) criteria, which is known to be nearly optimal when the SNR is high [11]. For each subchannel, we can choose at most $M$ users for which $\left\|\mathbf{w}_{n, k}\right\|^{2}>0$ and, for those users, the beamforming vectors must meet the orthogonality constraints $\mathbf{h}_{n, k} \mathbf{w}_{n, j}=0, j \neq k$. Under the ZF constraint, the inter-user interference term becomes zero in (1) and the achievable rate of user $k$ on subchannel $n$ is 
given by

$$
r_{n, k}\left(\mathbf{w}_{\mathbf{n}, \mathbf{k}}\right)=\log _{2}\left(1+\left\|\mathbf{h}_{n, k} \mathbf{w}_{n, k}\right\|^{2}\right) .
$$

The set of users $\mathcal{K}$ is divided into a set $\mathcal{D}$ of RT users with minimum rate constraints $\check{d}_{k}>0$ and a set $(\mathcal{K}-\mathcal{D})$ of non real-time $(\mathrm{nRT})$ users for which $\check{d}_{k}=0$. The user selection is modelled by the binary variables $\alpha_{k, n}$ which take the value 1 when the user $k$ is selected in the SDMA set of subchannel $n$, and zero otherwise.

The objective of the RA algorithm is to maximize the weighted sum rate of the users subject to the power, minimum rate and $\mathrm{ZF}$ constraints. The users weights $c_{k}$ and the minimum rate constraints are determined by a higher layer scheduler.

Defining $\mathbf{w}, \boldsymbol{\alpha}$ as the vectors of stacked optimization variables $\mathbf{w}_{n, k}, \alpha_{n, k}$, the RA problem can be mathematically formulated as follows:

$$
\begin{gathered}
\max _{\mathbf{w}, \boldsymbol{\alpha}} \sum_{n=1, k=1}^{N, K} c_{k} r_{n, k}\left(\mathbf{w}_{n, k}\right) \\
\sum_{n=1, k=1}^{N, K}\left\|\mathbf{w}_{n, k}\right\|^{2}-\check{P} \leq 0 \\
-\sum_{n=1}^{N} r_{n, k}\left(\mathbf{w}_{n, k}\right)+\check{d}_{k} \leq 0, \quad k \in \mathcal{D} \\
\sum_{k} \alpha_{n, k} \leq M, \quad \forall n \\
\left(\mathbf{h}_{n, k} \mathbf{w}_{n, j}\right)^{2} \leq B^{\prime}\left[\left(1-\alpha_{n, k}\right)+\left(1-\alpha_{n, j}\right)\right] \\
\forall n, \forall k, \forall j, k \neq j \\
\left\|\mathbf{w}_{n, k}\right\| \leq A^{\prime} \alpha_{n, k} \\
\alpha_{n, k} \in\{0,1\}
\end{gathered}
$$

Constraint (4) is the total power constraint imposed on the beamforming vectors and constraints (5) assure that the RT users are assigned rates larger or equal than their minimum rates $\check{\delta}_{k}$. Constraints (6) to (9) correspond to the ZF constraints: Eq. (6) guarantees that we do not choose more than $M$ users for each subchannel, Eq. (7) that two users in an SDMA set meet the ZF constraints and Eq. (8) that the beamforming vector is null for users that are not in an SDMA set, $A^{\prime}$ and $B^{\prime}$ are some large constants. 
Problem (36) is a non-linear mixed integer program (NLMIP). The vector of binary variables $\alpha$ determines the set of users that are assigned to each subchannel. On the other hand, the vector of continuous variables $\mathbf{w}$ determine the beamforming vectors and need to comply with the ZF constraints (7-8) which depend on the user selection binary variables $\boldsymbol{\alpha}$. There are many offthe-shelf software packages available to solve NLMIPs, see [12] for a survey. They use different methods with different levels of accuracy and speed. However, the current NLMIP solvers do not automatically exploit the specific structure of problem (3-9). An off-line method is proposed in [5] to solve this problem with near-optimality which we use to compare our heuristics.

\section{EFFICIENT HEURISTIC METHODS}

In this section, we propose heuristic methods to solve problem (3-9) efficiently. We are interested in feasible solutions, i.e., points that satisfy the rate and power constraints, and that are not too far from the optimal solution. In the dual-based near-optimal [5], power allocation and subchannel assignment are jointly performed. Except for some trivial cases, we cannot separate the subchannel allocation and power allocation processes. For heuristic methods, however, we separate these processes in order to reduce computational complexity. In the first stage, we find a subchannel assignment that has enough subchannels assigned to the real-time (RT) users, and in the second stage, we allocate power among users using the fixed subchannel assignment. For the subchannel assignment stage, we make use of the well known Semiorthogonal User Selection (SUS) algorithm [11] to select user channel vectors that have large norms and are semiorthogonal to each other. But contrary to the throughput maximization case, we include the RT users to satisfy their minimum rates when selecting the user set for each subchannel. For the power allocation stage, we use a method that finds feasible points and is much quicker than optimally solving the power optimization problem. The subchannel assignment algorithm and the power allocation algorithm constitute the proposed heuristic method.

\section{A. General Description of the Proposed Heuristic Method}

The basis for the design of our heuristic is the realization that for a fixed subchannel assignment $\boldsymbol{\alpha}$ in problem (3-9), the resulting power allocation problem can be approximated to a convex one which is much easier to solve. Thus, instead of enumerating all feasible values of variable $\boldsymbol{\alpha}$ corresponding to every subchannel assignment, our heuristic chooses a subchannel assignment 
and solves a convex power allocation problem. Then, it reassign resources and solves the power allocation problem again until the RT users' rate constraints are met.

There are two mechanisms to reassign resources to users. The first mechanism - subchannel reassignment - takes away subchannels assigned to users that do not require them, because they are not RT users or they have more resources than needed, and assigns them to the users in need. The second mechanism - rate-constrained power allocation - takes into account the user rate constraints to reallocate power between users. Subchannel reassignment has a much larger effect because users in need are given subchannels that they did not have before; the rates increase substantially with every subchannel added. Rate-constrained power allocation has a lower effect because the rate increase dependency against power is logarithmic. However, this mechanism proves to be crucial in finding feasible points when the minimum rate requirements increase. In addition, recomputing the users power is quicker than finding a new subchannel and inverting its new channel matrix.

The proposed heuristic method starts by solving problem (3-9) without considering rate constraints (5). If the required rates $\breve{d}_{k}$ are lower or equal than the obtained rates, we have an optimal solution and the algorithm finishes. To obtain the maximum throughput solution efficiently, we use the SUS algorithm to assign subchannels to users and then perform maximum throughput power allocation, which consists of finding the user power allocation that satisfies the power constraint with equality disregarding the rate constraints. These correspond to the first two blocks in the diagram of figure 1. If the required rates are met, we exit, otherwise, we need to assign more resources to the users in need, thus we perform rate-constrained power allocation as indicated by block 3 in figure 1

We perform subchannel re-assignment when the maximum throughput subchannel assignment plus rate-constrained power allocation does not support the required minimum rates. A heuristic method that groups semiorthogonal user vectors is used to assign more subchannels to the users in need and it is indicated by block 4 in figure 1. We perform iterations adding subchannels to users in need and performing power allocation (blocks 2 and 3) until the user minimum rates are met or there are no more subchannels to reassign and the problem is declared unfeasible by the heuristic. 


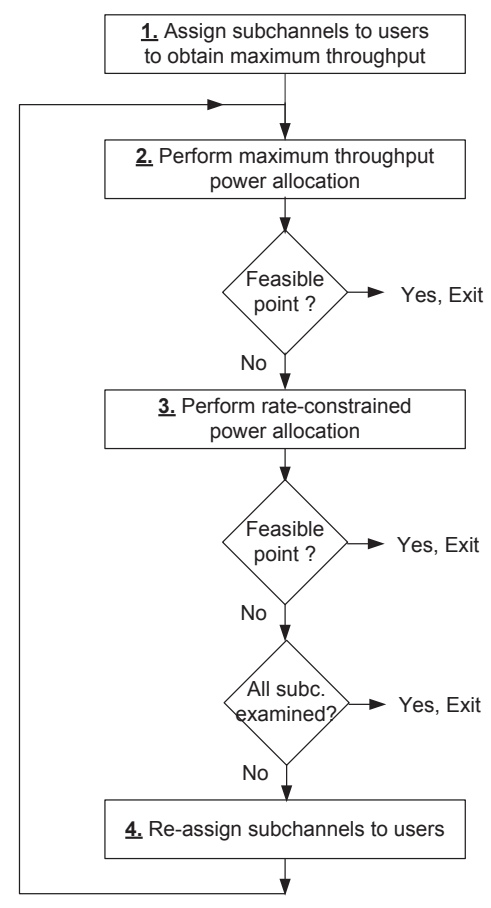

Fig. 1: Heuristic general algorithm

\section{B. Power Allocation}

In this subsection we find the user power allocation for a fixed subchannel assignment. Assume that we have chosen a vector $\boldsymbol{\alpha}$ for each subchannel $n$ satisfying Eqs. (6) and (9. We explain the heuristic method to obtain such a vector in section ЩII-E. The vector $\boldsymbol{\alpha}^{(n)}$ determines a fixed SDMA set of users, $S_{n}$, defined as

$$
\begin{aligned}
& S_{n} \doteq\left\{k \in \mathcal{K}: \alpha_{n, k}=1\right\}, \\
& g_{n} \doteq\left|S_{n}\right|, \quad \forall n .
\end{aligned}
$$

Sets $S_{n}$ contain the indexes of the users assigned to subchannel $n$. We first reformulate problem (3-19) using these known sets. Then, we apply a dual method to solve it. For this purpose, we arrange the channel vectors of selected users in the rows of a $g_{n} \times M$ matrix

$$
\mathbf{H}_{n} \doteq\left[\begin{array}{c}
\mathbf{h}_{n, S_{n}(1)} \\
\vdots \\
\mathbf{h}_{n, S_{n}\left(g_{n}\right)}
\end{array}\right], \quad \forall n,
$$


where $S_{n}(j)$ is the $j$-th user in the set $S_{n}$. We also arrange the corresponding beamforming vectors in the columns of a $M \times g_{n}$ matrix for each subchannel

$$
\mathbf{W}_{n} \doteq\left[\mathbf{w}_{n, S_{n}(1)}, \ldots, \mathbf{w}_{n, S_{n}\left(g_{n}\right)}\right], \quad \forall n .
$$

Then, the ZF constraints (7) can be written as

$$
\mathbf{H}_{n} \mathbf{W}_{n}=\operatorname{diag}\left(\sqrt{\mathbf{q}^{(n)}}\right), \quad \forall n
$$

where $\mathbf{q}^{(n)}=\left\{q_{n, j}\right\}$ is the users power vector comprised of

$$
q_{n, j}=\mathbf{h}_{n, s_{n}(j)} \mathbf{w}_{n, s_{n}(j)}, \quad j \in\left\{1, \ldots, g_{n}\right\}
$$

Beamforming vectors for users $k$ not belonging to $s_{n}$ are set to zero. Restricting the direction of $\mathbf{W}_{n}$ to the pseudo-inverse of matrix $\mathbf{H}_{n}$ as done in [5], we obtain from (14)

$$
\mathbf{W}_{n}=\mathbf{H}_{n}^{\dagger} \operatorname{diag}\left(\sqrt{\mathbf{q}^{(n)}}\right), \quad \forall n
$$

the power constraint can now be written as

$$
\sum_{n=1}^{N} \operatorname{tr}\left(\mathbf{W}_{n}^{H} \mathbf{W}_{n}\right)-\check{P} \leq 0
$$

and replacing (16) in (17) we obtain

$$
\sum_{n=1}^{N} \sum_{j=1}^{g_{n}}\left[\left(\mathbf{H}_{n}^{\dagger}\right)^{H} \mathbf{H}_{n}^{\dagger}\right]_{j, j} q_{n, j}-\check{P} \leq 0
$$

Let's define the entries of the $N \times K$ matrices $\boldsymbol{\beta}$ and $\mathbf{p}$ as

$$
\begin{aligned}
& \beta_{n, k} \doteq \begin{cases}{\left[\left(\mathbf{H}_{n}^{\dagger}\right)^{H} \mathbf{H}_{n}^{\dagger}\right]_{j, j}} & \text { if } k=s_{n}(j), \quad \forall j \in\left\{1, \ldots, g_{n}\right\} \\
0, & \text { otherwise }\end{cases} \\
& p_{n, k} \doteq \begin{cases}q_{n, j} & \text { if } k=s_{n}(j), \quad \forall j \in\left\{1, \ldots, g_{n}\right\} \\
0, & \text { otherwise. }\end{cases}
\end{aligned}
$$

The power constraint can now be expressed as

$$
\sum_{n=1}^{N} \sum_{k=1}^{K} \beta_{n, k} p_{n, k}-\check{P} \leq 0 .
$$


From the original model (3-9), we do not need constraints (6) and (9) because we choose $\alpha_{n, k}$ satisfying these conditions. Constraints (7) and (8) are implicit in the reformulated model because the beamforming vectors satisfy (14) and we set to zero all beamforming vectors for which $\alpha_{n, k}=0$. Therefore, only constraints (4) and (5) remain. We also changed the problem optimization variables from the vectors $\mathbf{w}_{n, k}$ to the scalars $p_{n, k}$ because the vector directions are now fixed by (16).

Replacing $\left(\mathbf{h}_{n, k} \mathbf{w}_{n, k}\right)^{2}$ by $p_{k, n}$ in (3),(5) and replacing (4) by (21), we obtain the problem formulation

$$
\begin{aligned}
& \max _{p_{n, k}} \sum_{n=1}^{N} \sum_{k=1}^{K} c_{k} \log _{2}\left(1+p_{n, k}\right) \\
& \sum_{n=1}^{N} \sum_{k=1}^{K} \beta_{n, k} \quad p_{n, k}-\check{P} \leq 0 \\
&-\sum_{n=1}^{N} \log _{2}\left(1+p_{n, k}\right)+\check{d}_{k} \leq 0, \quad k \in \mathcal{D} \\
& p_{n, k} \geq 0, \quad \forall n, k .
\end{aligned}
$$

\section{Optimal Power Allocation}

Problem (22-25) is convex since it maximizes a concave function over a convex set formed by constraints (23-25). We can solve this problem optimally using a dual Lagrange approach. First, we define dual variables $\theta$ for the power constraint (23), and $\left\{\delta_{k}\right\}$ for the rate constraints (24). Then, we derive a closed-form expression of the dual function and solve the dual problem. This yields the water-filling power allocation [13]

$$
\bar{p}_{n, k}=\left[\frac{c_{k}+\delta_{k}}{\theta \beta_{n, k} \ln 2}-1\right]^{+}, \quad \forall n, \forall k: \beta_{n, k} \neq 0
$$

and rate allocation

$$
r_{n, k}=\log _{2}\left(1+\left[\frac{c_{k}+\delta_{k}}{\theta \beta_{n, k} \ln 2}-1\right]^{+}\right), \quad \forall n,
$$

where $\delta$ is the vector of dual variables $\delta_{k}$. For convenience we have defined dual variables $\delta_{k}$ for all users including the ones with no minimum rate requirements $(k \notin \mathcal{D})$; for these users we set $\check{d}_{k}=0$.

We can the optimal dual variables $\theta,\left\{\delta_{k}\right\}$ using derivative-free techniques. The use of such methods involves two steps. In the first one, we compute a matrix pseudo-inverse per subchannel 
to obtain the inverse of the channel effective gains $\beta_{n, k}$; this has computational complexity $O\left(N M^{3}\right)$. In the second step, we perform subgradient iterations computing the power and rate constraints to obtain the subgradient vector; this has computational complexity is $O(N)$ $D) I_{d}$ ), assuming a maximum number of subgradient iterations $I_{d}$.

\section{Efficient Power Allocation}

To solve problem (22-25) more efficiently we separate it in two stages: maximum-throughput power allocation (PA) and rate-constrained PA. Maximum-throughput PA only considers the power constraint (23), thus $\delta_{k}=0$ in (26) and we just need to find the dual variable $\theta$ that satisfies the power constraint with equality. For this purpose, we use the exact method reported in [14], [15] which is summarized below

1) Find the dual variable $\theta$ that satisfies the power constraint (23) with equality, using

$$
\theta^{(i)}=\frac{\sum_{k=1}^{K}\left|\mathcal{B}_{k}\left(\check{\theta}, \delta_{k}\right)\right| c_{k}}{\left(\check{P}+\sum_{k=1}^{K} \sum_{n \in \mathcal{B}_{k}\left(\check{\theta}, \delta_{k}\right)} \beta_{n, k}\right) \ln 2},
$$

where $\check{\theta}>0$ is a lower bound of $\theta, \delta_{k}=0, i$ is the iteration index and

$$
\begin{array}{r}
\mathcal{B}_{k}\left(\theta, \delta_{k}\right) \doteq\left\{n \in \mathcal{N}:\left(k \in S_{n}\right) \wedge\right. \\
\left.\left(\beta_{n, k}<\frac{\left(c_{k}+\delta_{k}\right)}{\theta \ln 2}\right)\right\}, \quad \forall k \in \mathcal{K},
\end{array}
$$

with $S_{n}$ is the SDMA set associated to subchannel $n$, and $\mathcal{N}$ the set of all subchannels.

2) Recompute sets $\mathcal{B}_{k}^{(i+1)}$ using $\theta^{(i)}$. If the sets $\mathcal{B}_{k}^{(i)}$ and $\mathcal{B}_{k}^{(i+1)}$ are equal, we have found the solution, the power constraint is satisfied with equality. Otherwise, iterate recomputing (28) and (29) until finding identical sets in two consecutive iterations.

After solving the maximum throughput PA problem, if the achieved rates are feasible they correspond to the optimal ones. If the rate constraints are not met, we incorporate the rate constraints (24) and perform rate-constrained PA using the heuristic reported in [15]. This method finds a feasible point and does not require any iteration. This feasible point satisfies the power constraint with equality but the rate constraints with inequality and is faster to compute than the subgradient algorithm. Here, we summarize the method

1) Compute the set of unsatisfied users $\mathcal{T}$ as

$$
\mathcal{T} \doteq\left\{k \in\{1, \ldots, K\}: r_{k}<\check{d}_{k}\right\}
$$




$$
r_{k} \doteq \sum_{n=1}^{N} \log _{2}\left(1+\left[\frac{c_{k}}{\theta^{(1)} \beta_{n, k} \ln 2}-1\right]^{+}\right)
$$

where $\theta^{(1)}$ is the optimal power allocation dual variable obtained after maximum throughput PA.

2) For the users that do not belong to $\mathcal{T}$, make $\delta_{k}=0$. For the other users, obtain the minimum value of the dual variable $\delta_{k}$ required to satisfy the rate constraints

$$
\delta_{k}^{(2)}=\left[(\bar{\theta} \ln 2)\left(2^{\check{d}_{k}} \prod_{n \in \mathcal{A}_{k}^{\prime}} \beta_{k, n}\right)^{\left|\mathcal{A}_{k}^{\prime}\right|^{-1}}-c_{k}\right]^{+}
$$

where $\mathcal{A}_{k}^{\prime}=\mathcal{B}_{k}\left(\bar{\theta}, \delta_{k}\right), \bar{\theta}$ is an upper bound of $\theta$ given by

$$
\bar{\theta}=\theta^{(1)} 2^{\left(\breve{d}_{k}-r_{k}\right) \epsilon}
$$

and $\epsilon>0$ is a parameter found experimentally as explained in subsection IV-A.

3) Using dual variables $\delta_{k}^{(2)}$, compute the power constraint dual variable $\theta$ that satisfies the constraint (23) with equality.

Maximum throughput and rate constrained power allocation correspond to blocks 2 and 3 in figure 1.

\section{E. Subchannel Assignment Heuristic}

The purpose of block 1 in figure 1 is to perform user subchannel assignment to obtain high rates. The rates in (27) are affected by the effective channel gains $\beta_{n, k}^{-1}$, which increase when the channel vector norms are large and the chosen vectors for each subchannel are semi-orthogonal to each other. We use the well-known SUS algorithm [11] to perform this assignment. The computing efficiency of this algorithm is improved in [16]. We rewrite the SUS algorithm splitting it in two parts: an initialization stage and a user search stage. This is done to adapt the SUS algorithm to the rate-constrained case described in subsection III-F

The input to SUS initialization stage is the set of available users $\mathcal{U}_{0}$. Its output are the user with the maximum norm $\left\{\pi_{0}\right\}$ and a matrix $\mathbf{G}_{0}$ forming a basis of the null space spanned by the channel vector $\mathbf{h}_{\pi_{0}}$. We write the input/output relation of this stage as $\left[S_{n}^{0}, \mathbf{G}_{n}^{0}\right]=$ SUS_init $\left(\mathcal{U}_{0}\right)$, where $S_{n}^{0}$ contains only the selected first user $\left\{\pi_{0}\right\}$.

In the SUS search stage, we compute the projection of the remaining channel vectors to the null space spanned by the channel vectors of the users already selected. We pick the user whose 
projection is the largest, add it to set $S_{n}$ and recompute matrix $\mathbf{G}_{n}$. We add users until the set contains $M$ users or we finish examining all users in the input set $\mathcal{U}_{0}$. The input/output relation of the users search stage is $\left[S_{n}, \mathbf{G}_{n}\right]=$ SUS_search $\left(\mathcal{U}_{0}, S_{n}^{0}, \mathbf{G}_{n}^{0}\right)$, where $S_{n}$ is the set of selected users for subchannel $n$ and $\mathbf{G}_{n}$ its the matrix that spans the null space of the selected user vectors. The SUS algorithm as described in [11] is implemented by making $\mathcal{U}_{0}=\{1, \ldots, K\}$ and sequentially invoking the two stages: $\left[S_{n}^{0}, \mathbf{G}_{n}^{0}\right]=$ SUS_init $\left(\mathcal{U}_{0}\right)$ and $\left[S_{n}, \mathbf{G}_{n}\right]=$ SUS_search $\left(\mathcal{U}_{0}-S_{n}^{0}, S_{n}^{0}, \mathbf{G}_{n}^{0}\right)$.

After performing subchannel assignment for each subchannel $n$, we perform maximum throughput and rate constrained power allocation — indicated by blocks 2 and 3 in figure 1 - using the heuristics described in $\amalg I-D$.

\section{F. Subchannel Reassignment Heuristic}

After executing block 3 of the diagram in figure 1 , we obtain the user rates per subchannel $r_{n, k}^{(0)}$. The user rates are simply computed by $r_{k}^{(0)}=\sum_{n \in \mathcal{C}_{k}} r_{n, k}^{(0)}$, where $\mathcal{C}_{k}$ is the set of subchannels assigned to user $k$. We compute the set of unsatisfied users $\mathcal{T}$ given by (30). Set $\mathcal{T}$ tells us which users need to be assigned additional subchannels. We first scan the subchannels in which any of the users in need have good channel conditions, so they can be first reassigned to these users. The computational complexity of the subchannel ordering is bounded by $O\left(N^{2}\right)$. For each subchannel $n$, we build a critical set $\mathcal{E}$ containing the users in the current SDMA set that can not be removed from the SDMA set because that would take the user out of feasibility. We consider two cases: first that the set $\mathcal{E}$ is empty. In this case we invoke the SUS initialization algorithm to select the strongest user in $\mathcal{T}$ as the first element of the SDMA set. Then, we invoke the SUS search stage to add users to the SDMA set. We initially scan other users in $\mathcal{T}$ so that they can be added with priority to the SDMA set, and if the SDMA set has not yet been completed, we scan the remaining users $\{1, \ldots, K\}-\mathcal{T}$.

In the second case, when set $\mathcal{E}$ is not empty, we initialize the SDMA set with all the users in set $\mathcal{E}$, and then add users invoking the SUS search algorithm. To add users to this SDMA set, we scan the rest of the users but look first in the set of users in need $\mathcal{T}$. The difference between the cases $\mathcal{E}=\emptyset$ and $\mathcal{E} \neq \emptyset$ is that in the second case, we keep the users in need that are already in the SDMA set before trying to add more users. 


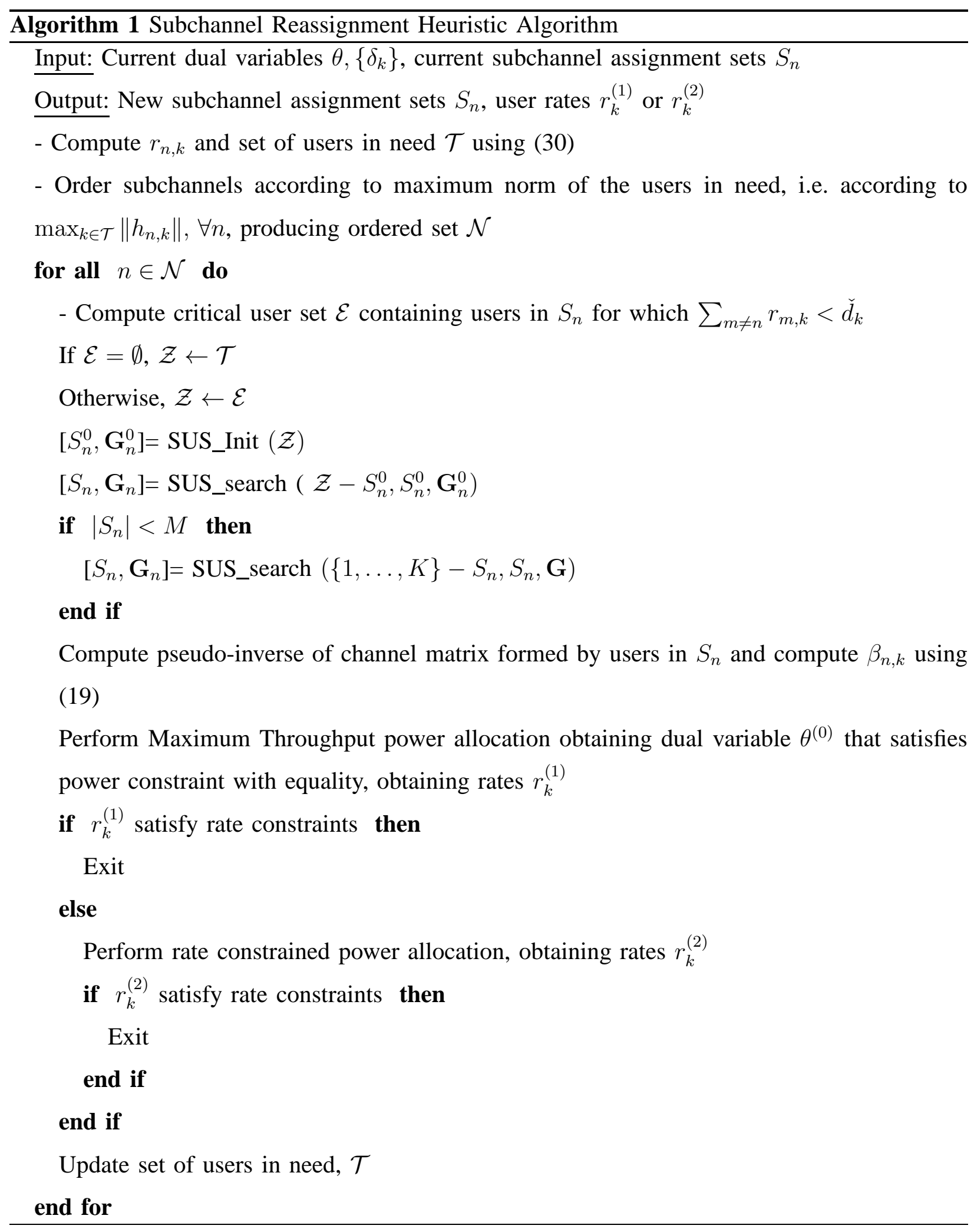




\begin{tabular}{|c|c|c|}
\hline Algorithm & Complexity & Purpose \\
\hline Alg. 1 & Eq. 34 & Prob. 39 \\
\hline Alg. 2 & $O\left(K N M^{3}\right)$ & $\begin{array}{c}\text { Prob. } 3 \text { with } \\
\text { per-subchannel power } \\
\text { constraint }\end{array}$ \\
\hline $\begin{array}{c}\text { Papoutsis' method } \\
\text { 99 }\end{array}$ & $O\left(K N^{2} M^{4}\right)$ & $\begin{array}{c}\text { Prob. } 3 \text { with } \\
\text { per-subchannel power } \\
\text { constraint }\end{array}$ \\
\hline Dual bound [5] & $O\left(N K^{M} M^{3}\right)$ & Dual of 3 \\
\hline
\end{tabular}

TABLE I: Algorithms complexity

Notice that all the selected users comply with the semiorthogonality condition of the SUS algorithm; the only change in computations to the SUS algorithm is the order in which we examine the users. By changing the order, we are giving priority to the users in need. The computational complexity of this stage is bounded by the complexity of the maximum throughput SUS search algorithm, i.e. $O\left(K M^{3}\right)$.

After obtaining the new SDMA set for a subchannel, we perform maximum throughput power allocation. If the resulting rates are feasible we exit the algorithm. Otherwise, we perform rateconstrained power allocation. If the resulting rates are still not feasible, we continue reassigning subchannels until the rates are feasible or there are no more subchannels and the algorithm declares that is not able to find a feasible point. This corresponds to the loop in the lower part of the block diagram in figure 1. The pseudo-code of the subchannel reassignment heuristic is listed in algorithm 1, which corresponds to the sequence of blocks 4,2,3 in figure 1,

The power allocation algorithms have computational complexity $O(K N)$ [15] and the SUS search algorithm has complexity $O\left(K M^{3}\right)$. Assuming the worst case where all subchannels are examined for reassignment, the proposed algorithm's overall computational complexity is

$$
O_{\text {alg. [1] }}= \begin{cases}O\left(K N^{2}\right), & \text { if } N>M^{3} \\ O\left(K N M^{3}\right), & \text { otherwise. }\end{cases}
$$

This is lower than Papoutsis' method [9] computational complexity, which is $O\left(K N^{2} M^{4}\right)$ for all $N$. 


\section{G. Reduced Complexity Algorithm}

In this section, we devise a variation to the subchannel reassignment algorithm 1 that linearizes the dependency of the computational complexity in expression (34) with respect to the number of subchannels $N$, for $N>M^{3}$. Since in LTE-Advanced systems, the maximum number of subchannels is large, it is important to linearize the computational complexity with respect to $N$.

For this purpose, we solve a sum rate maximization problem with one power constraint per subchannel instead of a total power constraint and we do not consider the rate constraints (24). In the subchannel iteration loop in algorithm 1, we update the user power corresponding to all subchannels because the power-constraint dual variable affects them all, which produces the term $N^{2}$ in the complexity expression (34) for $N>M^{3}$. To solve the new problem formulation, we need to update the user power corresponding to only one subchannel. Therefore, when computing the power and rates after power allocation, only the ones corresponding to that subchannel are affected, making the computational complexity of this step $O(K)$ as opposed to the original $O(K N)$. The computational complexity of this method is

$$
O_{\text {alg. } 2}=O\left(K N M^{3}\right)
$$

(35) varies linearly with $N$ and since $N$ ranges from 6 to 550 in a LTE-Advanced system with Carrier Aggregation (CA) [3], this results in a much faster algorithm for large $N$. The reduced complexity method does not support the high minimum rates that algorithm 1 can, but it is a more efficient algorithm when $N$ is large. We name this simplified method algorithm 2 , but we do not provide its pseudo-code since the differences with algorithm 1 are straightforward. Table I summarizes the computational complexity of the methods presented in this paper.

\section{NumericAl EVAluations}

In this paper we devised heuristic algorithms to efficiently solve problem (3-9). For this purpose, we proposed algorithm 1 in subsection $\amalg I I-F$ and its simplified version, algorithm 2 , in subsection $\amalg$ II-G. Table $\amalg$ shows that they have reduced computational complexity when compared to other methods. In this section, we numerically evaluate their performance and CPU load, and compare them against existing methods. The power allocation (PA) heuristics presented in subsection $\amalg$ II-D are invoked at each subchannel assignment iteration as illustrated in the block 
diagram of figure 1. Thus, the CPU load and performance of the PA algorithms have an important effect on the overall heuristic performance. In subsection IV-A we evaluate the performance of these PA heuristics independently of the subchannel assignment method, we assume certain subchannel assignment and evaluate the PA heuristics.

In subsection IV-B we evaluate the performance of the proposed overall heuristics and the support of the fulfilled minimum rates. Our interest is on answering the following questions: how far from the optimal is the sum rate achieved by these methods; how fast are the proposed heuristics compared to existing methods; what is the range of the minimum rates supported by the rate-constrained PA methods, as opposed to the maximum throughput PA methods; and how the overall heuristics compare to [9].

\section{A. Performance of Power Allocation Heuristics}

Figure 2 shows an example of the performance obtained by the PA heuristics presented in subsection [II-D for the listed system parameters. In the figure, we compare the sum rate given by the optimal solution to problem $(22-\sqrt{25})$ and the result given by the PA heuristics. For minimum rate constraints lower than $47 \mathrm{bps} / \mathrm{Hz}$, the rate constraints are inactive and the maximum throughput PA heuristic method gives the optimal sum rate. However, for minimum rate constraints higher than $47 \mathrm{bps} / \mathrm{Hz}$, the performance of the rate-constrained PA heuristic is sub-optimal and it depends on the parameter $\epsilon$ in (33). From the results of figure 2 , we observe that a parameter value close to $\epsilon=0.2$ achieves a large minimum rate support. For the example in figure 2, rate-constrained PA extends the range of the minimum rates constraints from 47 to $56 \mathrm{bps} / \mathrm{Hz}$, i.e., $16 \%$. Evaluating the performance over multiple configurations, we find that rateconstrained PA increases the range of supported minimum rates between 15\% and 30\% when compared to schemes that perform maximum throughput PA only. In general, any subchannel assignment method can increase the supported minimum rates by performing rate-constrained PA as we show below.

1) Benefits of rate-constrained power allocation: Papoutsis method [9] only performs maximum throughput PA. If we add rate-constraints PA to Papoutsis method, we would achieve and increase in the range of minimum rate constraints supported. This is illustrated in figure 3 for the system parameters listed, where the minimum rates support is extended from $40 \mathrm{bps} / \mathrm{Hz}$ to $50 \mathrm{bps} / \mathrm{Hz}$, i.e., a $20 \%$ increase. In this example, we optimally solve a power allocation problem 


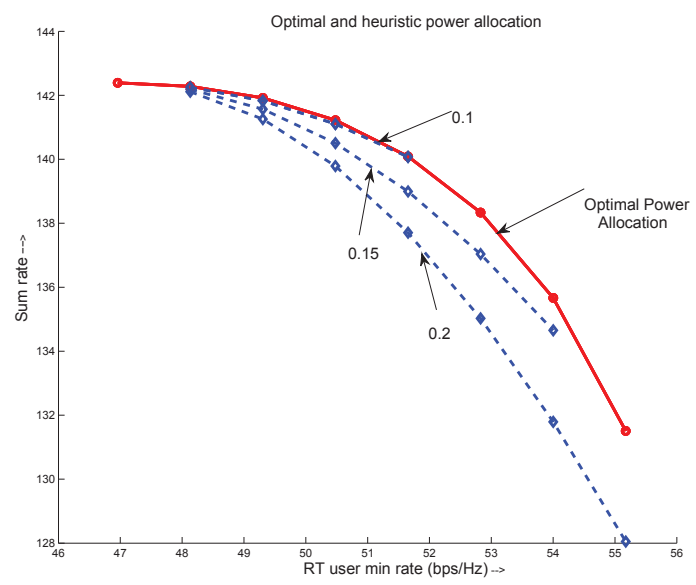

Fig. 2: Optimal and heuristic power allocation comparison for different values of $\epsilon . K=3, M=$ $3, N=1, \check{P}=20$.

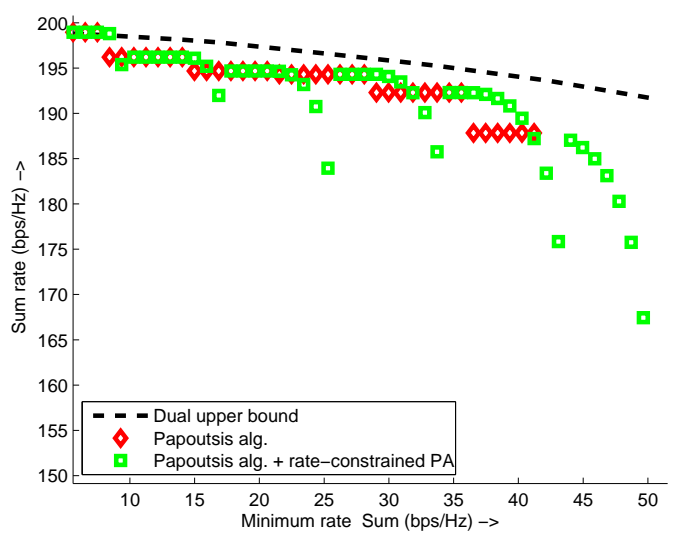

Fig. 3: Optimal and heuristic methods comparison. $K=16, M=3, N=8, D=1, \check{P}=20$.

similar to (22, 25), but with sub-channel power constraints instead of a total power constraint. The smearing effect in figure 3 occurs when the rate constraints are not satisfied with simple maximum throughput PA and rate-constrained PA is applied.

It is not possible, however, to solve the rate-constrained PA problem without an increase in the computational complexity. For this reason, we use the PA heuristics described in section III-D.

\section{B. Overall Heuristics Performance Comparison}

We use a Rayleigh fading channel model to generate independent channels and compare numerically the supported minimum rates and the sum rate achieved by the following methods: 


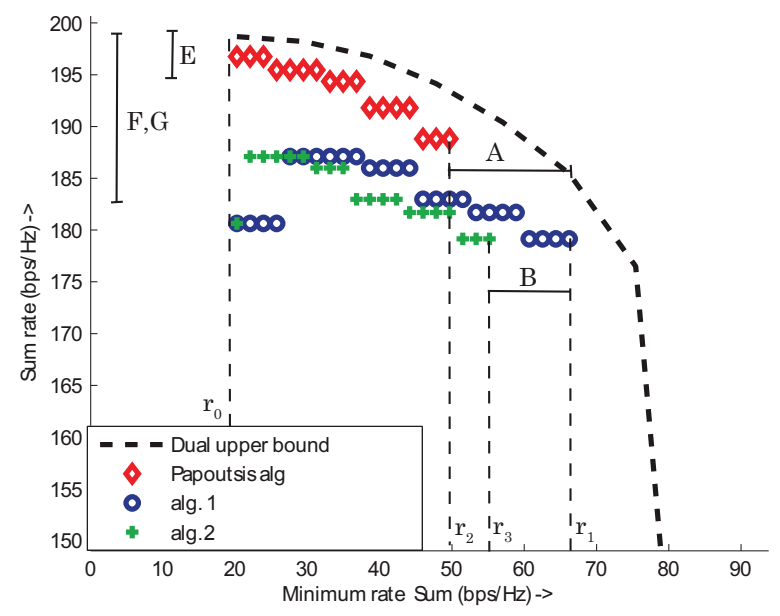

Fig. 4: Optimal and heuristic methods comparison.

1) Dual-based upper bound [5]

2) Papoutsis' algorithm [9]

3) The proposed heuristic SUS-based heuristic algorithm 1 described in subsection $\amalg$

4) A simplified SUS-based heuristic algorithm 2, described in subsection III-G that performs PA considering per-subchannel power constraints.

Figure 4 illustrates one example of the objective achieved by these methods for one real-time user, $D=1$ and parameters $N=8, K=8, M=3, \check{P}=20$. The plots only show feasible points. Thus, when increasing the minimum required rates (horizontal axis), the curves stop if the methods can no longer find feasible points.

We start by solving the problem without considering minimum rate requirements as indicated by blocks 1 and 2 in figure 1, this gives us user rates $\left\{r_{k}^{0}\right\}$. If we were to extend the curves of figure 4 to zero, they would be flat curves with $\sum_{k} r_{k}^{0}$ as the sum rate. We want to focus on the domain where rate constraints are active. For this purpose, we increase the rate constraints by increments $\Delta_{r}$, i.e., $\check{d}_{k}=r_{k}^{0}+\Delta_{r}$, for the RT users and solve the problem for each $\check{d}_{k}$.

For a number of RT users $D>1$, we use $\sum_{k=1}^{D} \check{d}_{k}$ to list the minimum rate constraint in plots. In our numerical evaluations, we increase the rate constraints and try to find feasible points using the heuristics until the dual upper bound becomes positive indicating the problem unfeasibility. In figure 4 the upper bound provided by the negative of the dual function maximum is shown by a dashed line and it is the reference to measure the performance of all heuristic 
methods. Papoutsis' method is shown in diamond markers and closely follows the upper bound. The proposed SUS-based heuristic (algorithm 1) is shown in circle markers and its simplified version (algorithm 2) in cross markers. They have lower performance than Papoutsis' method, but they increase the range of supported minimum rates. To quantify these observations, we define the following measurements:

- $A$ : The difference in percentage between the minimum rate supported by the SUS-based heuristic algorithm $1 r_{1}$, and Papoutsis' method $r_{2}$, i.e., $A=100\left(r_{1}-r_{2}\right) / r_{1}$.

- $B$ : The difference in percentage between the minimum rate supported by the SUS-based heuristic algorithm $r_{1}$ and its simplified version $r_{3}$ in percentage. $A$ and $B$ indicate how much the proposed SUS based algorithm 1 increases the range of supported minimum rates. The larger these measurements are, the better the proposed algorithm 1 .

- $E$ : The difference in percentage between the upper dual bound $u_{1}$ and the sum rate achieved by Papoutsis' method $u_{2}$, i.e. $E=100\left(u_{1}-u_{2}\right) / u_{1}$. To compute $u_{1}$ and $u_{2}$ we average the sum rates over the minimum rates supported by Papoutsis' method. This corresponds to rates between $r_{0}$ up to $r_{2}$ in figure 4 , where $r_{0}$ is sum of of the rates at which the $D$ RT users' rate constraints become active. We average the sum rates over the same rate interval for all methods.

- $F$ : The difference in percentage between the upper dual bound and the SUS-based heuristic algorithm 1 ,

- $G$ : The difference in percentage between the upper dual bound and the simplified SUSbased heuristic algorithm 2. E, $F$ and $G$ indicate how far the sum rate is from the upper bound for each method. The smaller this measurement is, the better the algorithm.

Averaging these measurements over 100 channel realizations, we obtain the results shown in figure 5 for various number of RT users, $D$. The difference, E, between Papoutsis' method performance and the upper bound is very small $(<2.5 \%)$. This is because Papoutsis' method minimizes the throughput reduction by scanning over all possible users swapping. The proposed heuristic methods have similar performance gaps against the dual bound ( $E, F \approx 13 \%$ ), which are larger than Papoutsis' method. However, they achieve this performance with a much lower computational complexity as we shall see. In addition, the proposed SUS-based heuristic method with rate-constrained power allocation, supports up to $20 \%$ larger minimum rates than the other 

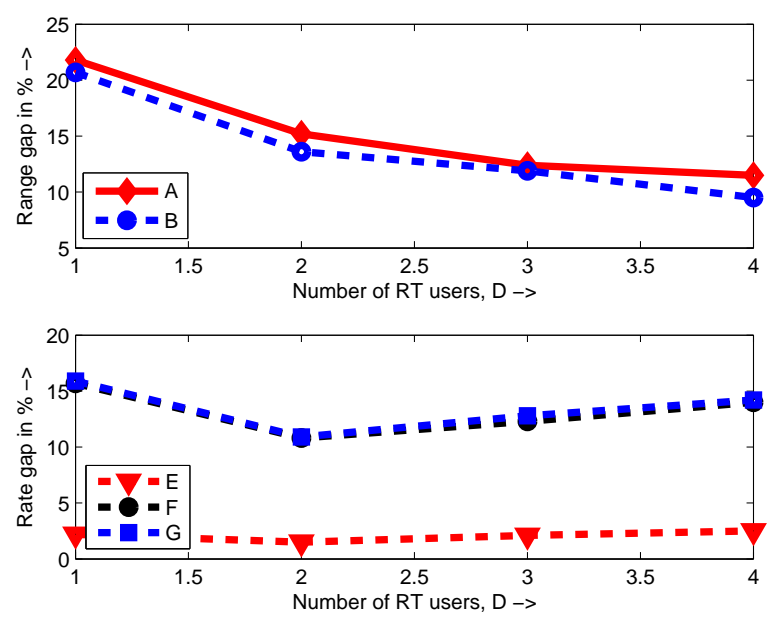

Fig. 5: Optimal and heuristic methods comparison vs. D.

two methods (see $A, B$ in figure 5). As the number of RT users $D$ increases, the difference $A$ decreases since it is harder for algorithm 1 to find feasible points. Recall that we force all $D$ user rate constraints to be active.

Figure 6 shows the results when varying the number of users $K$ from 8 to 32 . The performance of the proposed methods improves as the number of users increase, as indicated by the difference between the upper dual bound and the sum rate attained (measurements $F$ and $G$ decrease from $14 \%$ to $7 \%$ ). This is because in the presence of more users, the SUS algorithm is more likely to find semiorthogonal channel vectors, thus increasing the rates and effectively exploiting multiuser diversity. In contrast, Papoutsis' method slightly deteriorates when the number of users increase (measurement $E$ increases to $3.8 \%$ ).

\section{Increasing the number of RT users}

We now study an interesting scenario where we increase the number of RT users but keep fixed the minimum rates. This will correspond to the case of finding the maximum number of supported RT users (e.g. video) in a cell. We activate the rate constraints by setting the minimum rates to $10 \%$ more of the rates achieved by maximum throughput channel and power allocation.

Figure 7 shows the upper dual bound in dashed lines, the objective achieved by Papoutsis' method in star markers and the proposed heuristics in circle and square markers, as a function of the number of RT users. Papoutsis' method performance is very close to the upper bound, but it quickly degrades when the number of RT users increases. It cannot find feasible points 

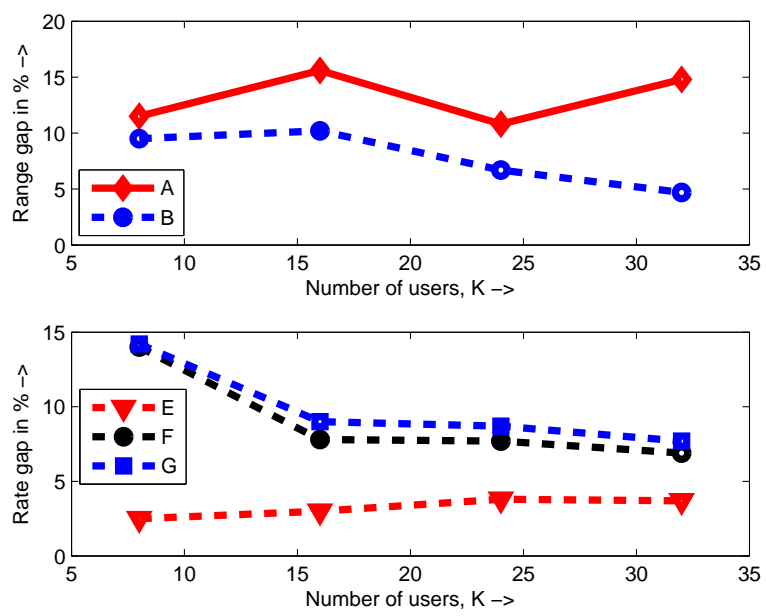

Fig. 6: Optimal and heuristic methods comparison vs. $K$.

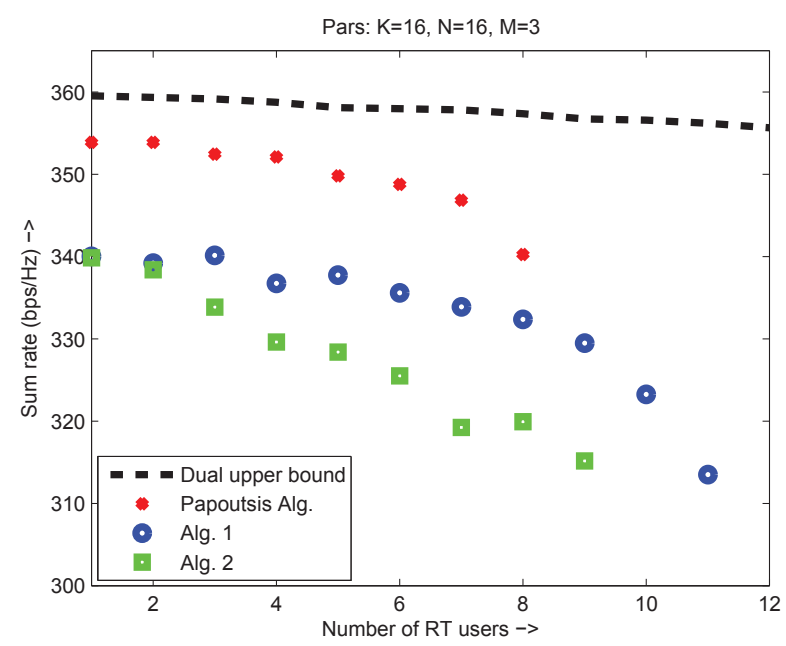

Fig. 7: Algorithms performance vs. the number of RT users

with 9 RT users onward, while the proposed heuristics yield solutions for these values within $12 \%$ of the upper bound.

Figure 8 shows the elapsed time employed by these heuristics. Papoutsis' method elapsed time grows approximately linear with the number of RT users and it is larger than both algorithms 1 and 2. This is because when the number of RT users increases, Papoutsis' method need to examine more combination of users and invert their corresponding channel matrices. Algorithms 1 and 2 grow much slower. According to figures 7 and 8 , not only algorithms 1 and 2 support a higher number of RT users than Papoutsis' method, but they require less computation time. 


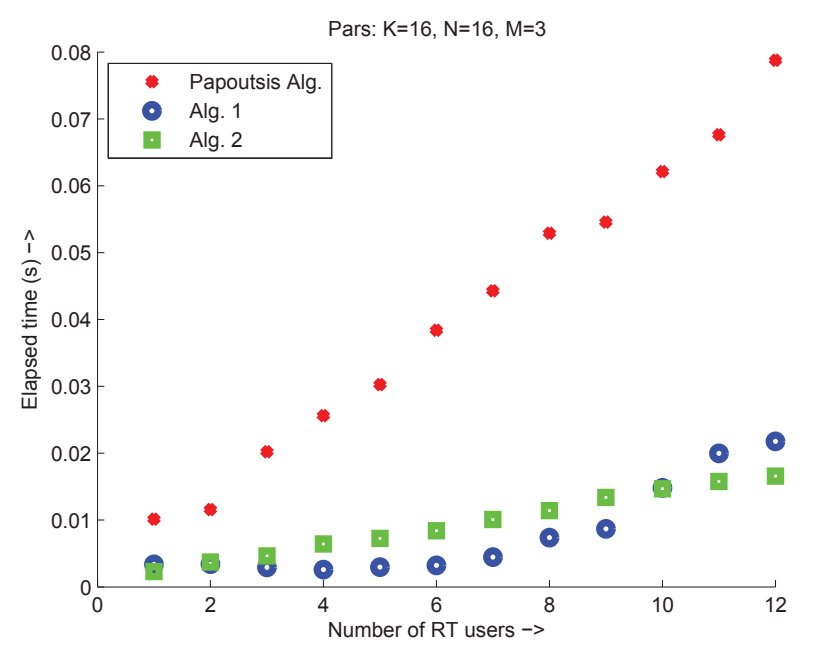

Fig. 8: Elapsed time vs. the number of RT users

\section{CONCLusions}

In this paper we designed algorithms to provide solution points to the RA problem for ZF beamforming MISO-OFDMA systems supporting minimum rate requirements. The solution points given by these algorithms differ in their distance to the optimal solution and in the computational complexity to obtain them. We designed two heuristic methods: algorithms 1 and 2, they select an SDMA set for each subchannel and then solve a power allocation problem. The difference between algorithms 1 and 2 is that the latter one considers power constraints per subchannel, providing a smaller minimum rates range but higher computational efficiency.

We showed through numerical evaluations that they have a performance not far from the optimal solution and that they increase the range of supported minimum rates when compared with other approach reported in the literature. This is an important result because, in a system with RT users, it is more important to satisfy the rate constraints of the users in need than increasing the rates of the nRT users. Compared with the method proposed in [9], our methods do not follow the upper bound as closely but they increase the range of the minimum rates supported. This and the fact that we have reduced the computational complexity, are the main advantages of the proposed methods. We also showed that rate constrained power allocation extends the range of the minimum rates supported when applied to other subchannel assignment methods. 


\section{REFERENCES}

[1] Cisco. (2013, Nov.) Global mobile data traffic forecast update, 20122017. Cisco. [Online]. Available: http://www.cisco.com/en/US/solutions/collateral/ns341/ns525/ns537/ns705/ns827/white_paper_c11-520862.html

[2] CRTC and I. Canada. (2013, Jan.) Price Comparisons of Wireline, Wireless and Internet Services in Canada, 2011 update. CRTC and Industry Canada. [Online]. Available: http://www.crtc.gc.ca/eng/publications/reports/rp1106.htm

[3] 3GPP, "3GPP: Evolved Universal Terrestrial Radio Access (E-UTRA); Base Station radio transmission and reception; Further advancements for E-UTRA physical layer aspects,” 3GPP TR V10.5, Tech. Spec.n Group Radio Access Network.

[4] IEEE, "IEEE Standard for Local and metropolitan area networks - part 16: Air Interface for Broadband Wireless Access Systems, 2011," Standard IEEE P802.16m, Institute of Electrical and Electronic Engineers.

[5] D. Perea-Vega, A. Girard, and J.-F. Frigon, "Dual-based bounds for resource allocation in zero-forcing beamforming OFDMA-SDMA systems,” EURASIP Journal on Wireless Communications and Networking, vol. 2013, no. 1, p. $51,2013$.

[6] C. Tsai, C. Chang, F. Ren, and C. Yen, "Adaptive radio resource allocation for downlink OFDMA/SDMA systems with multimedia traffic," IEEE Transactions on Wireless Communications, vol. 7, no. 5, pp. 1734-1743, 2008.

[7] W. Chung, L. Wang, and C. Chang, "A low-complexity beamforming-based scheduling for downlink OFDMA/SDMA systems with multimedia traffic," in Proc. of IEEE GLOBECOM, Nov. 2009, pp. 1-5.

[8] S. K. V. Papoutsis, I. Fraimis, "User selection and resource allocation algorithm with fairness in MISO-OFDMA," IEEE Communications Letters, vol. 14, no. 5, pp. 411-413, 2010.

[9] V. Papoutsis and S. Kotsopoulos, "Resource Allocation Algorithm for MISO-OFDMA Systems with QoS Provisioning," in Proc. ICWMC, The Seventh International Conference on Wireless and Mobile Communications, Jun. 2011.

[10] W. Lu, F. Ji, and H. Yu, "A General resource allocation algorithm with fairness for SDMA/MISO/OFDMA systems," Communications Letters, IEEE, vol. 15, no. 10, pp. 1072-1074, 2011.

[11] T. Yoo and A. Goldsmith, "On the optimality of multiantenna broadcast scheduling using zero-forcing beamforming," IEEE Journal on Selected Areas in Communications, vol. 24, no. 3, pp. 528-541, Mar. 2006.

[12] M. Bussieck. (2012, Oct.) MINLP solver software. Humboldt Universitat zu Berlin. [Online]. Available: http://www.math.hu-berlin.de/ stefan/minlpsoft.pdf

[13] D. Perea-Vega, A. Girard, and J.-F. Frigon, "Fast heuristic for power allocation in zero-forcing OFDMA-SDMA systems with minimum rate constraints," In preparation, vol. 2013, 2013.

[14] D. Perez-Palomar and J. Rodriguez-Fonollosa, "Practical algorithms for a family of waterfilling solutions," IEEE Transactions on Signal Procesing, vol. 53, no. 2, pp. 686-695, 2005.

[15] D. Perea-Vega, J. Frigon, and A. Girard, "Fast Heuristic for Power Allocation in Zero-forcing OFDMA-SDMA Systems," in - In preparation, available on-line at www.arxiv.org/abs/...

[16] J. Mao, J. Gao, Y. Liu, and G. Xie, "Simplified Semi-Orthogonal User Selection for MU-MIMO Systems with ZFBF," IEEE Wireless Communications Letters, vol. 1, no. 1, pp. 42-45, 2012. 